\title{
Investigation of Medication Errors: A Prescription Survey from Sri Lanka
}

\author{
KIM De Silva ${ }^{1}$, KPRC Parakramawansha ${ }^{2}$, SHT Sudeshika ${ }^{1}$, Chaminie B \\ Gunawardhana $^{1}$ and MHF Sakeena ${ }^{3 *}$ \\ ${ }^{1}$ Department of Pharmacy, Faculty of Allied Health Sciences, ${ }^{2}$ Department of Pharmacology, Faculty of Medicine, University of \\ Peradeniya, Peradeniya, Sri Lanka, ${ }^{3}$ Faculty of Pharmacy, University of Sydney, New South Wales, Australia
}

${ }^{\star}$ For correspondence: Email: sakeenab22@yahoo.co.in; Tel: +94-77-9864620

\begin{abstract}
Purpose: To identify and quantify possible errors in handwritten outpatient prescriptions in relation to adherence to standard guidelines on the layout and content of prescriptions.

Method: A sample of 200 handwritten outpatient prescriptions were collected from two pharmacies located in a sub-urban (Aluthgama) and an urban (Kandy) area in Sri Lanka. Data were extracted using a pilot-tested questionnaire and the legibility of the prescription was assessed by three independent investigators. The results from the suburban area were compared with those from the urban area.

Results: Based on the layout of the prescription, the presence of patient information was unsatisfactory. Patient name and age were present in less than half of the prescriptions. However, prescriber information except registration number was present in more than $75 \%$ of the prescriptions. Date of consultation was present in $>81.5 \%$ of the prescriptions. Non-standard abbreviations were used in 36.5 $\%$ of the prescriptions while incomplete units were observed in $51 \%$ of the prescriptions. Nearly half of the prescriptions from both urban and suburban locations were illegible. Occurrence of prescriber details was a significantly different between Aluthgama and Kandy.

Conclusion: Prescription errors are common in outpatient settings of Aluthgama and Kandy areas in Sri Lanka. Standardized prescription writing process in relation to layout, use of abbreviations, and units and legibility, is proposed as a potential solution to overcome this problem.
\end{abstract}

Keywords: Medication error, Prescriptions, Standardized prescription writing, Prescriber information, Non-standard abbreviation

Tropical Journal of Pharmaceutical Research is indexed by Science Citation Index (SciSearch), Scopus, International Pharmaceutical Abstract, Chemical Abstracts, Embase, Index Copernicus, EBSCO, African Index Medicus, JournalSeek, Journal Citation Reports/Science Edition, Directory of Open Access Journals (DOAJ), African Journal Online, Bioline International, Open-J-Gate and Pharmacy Abstracts

\section{INTRODUCTION}

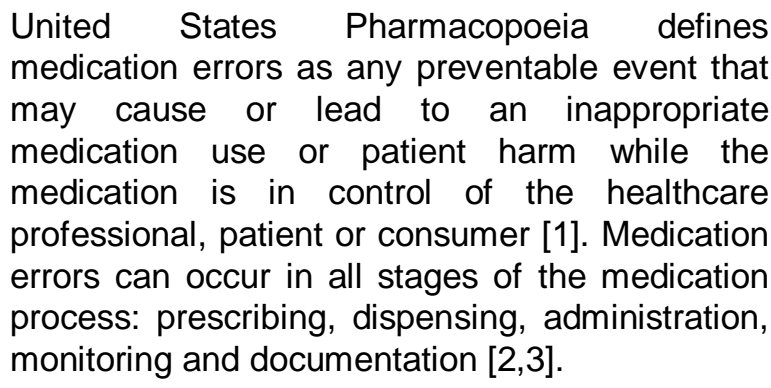

United States Pharmacopoeia defines medication errors as any preventable event that may cause or lead to an inappropriate medication is in control of the healthcare professional, patient or consumer [1]. Medication errors can occur in all stages of the medication monitoring and documentation $[2,3]$.
A study in USA stated that the medication errors are the eighth most frequent cause of death in USA which was more frequent than car accidents, breast cancers or AIDS [4]. Medication errors increase health care cost, increase unnecessary diagnostic tests and treatments, prolong hospital stay, decrease confidence of patients in health care system and increase the risk of patient death or serious patient morbidity almost two-fold [5-7]. 
Prescription error is a type of medication error which is defined as an error in prescribing decision or prescription writing process, where there is an unintentional significant reduction in the probability of treatment being timely and effective or increase in the risk of harm when compared with generally accepted practice [8]. Errors in prescribing decision can be subdivided as inappropriate prescription and pharmaceutical issues.

Errors in prescription writing process can be subdivided into failure to communicate essential information and transcription errors [8].

Prescription writing stage is the most common stage of errors $(99.12 \%)$ according to a study carried out in Indonesia on outpatients of a government hospital [9]. Another prospective observational study done in United Kingdom showed that $10 \%$ of prescriptions in hospitals contained errors [10].

This current study was conducted to assess the possible errors in outpatient handwritten prescriptions in relation to adherence to standard guidelines on layout and content of the prescriptions; use of abbreviations for drug name, use of non-standard abbreviations for directions for drug, use of incomplete units, use of decimal points and to evaluate legibility of prescriptions.

\section{EXPERIMENTAL}

\section{Setting}

Prescription errors were assessed using handwritten prescriptions. A convenient sample of two hundred (200) prescriptions were collected from two community pharmacies situated in two provinces, Western province and Central province during 4 months period from April 2013 to August 2013. One pharmacy is situated in Aluthgama which is a suburban area in Kalutara district of Western Province and the other one is in Kandy, an urban area of Central Province. Data were collected using a pilot tested questionnaire.

\section{Ethical issues}

Ethical approval was obtained from Ethical Review Committee of Faculty of Allied Health Sciences, University of Peradeniya, Peradeniya, Sri Lanka before commencing the study. Photos (snap shots) of the prescriptions without the information revealing patient and prescriber identities were taken after getting the verbal informed consent from the patient.

\section{Study design}

As the first part of the study, errors in the layout of the prescriptions were assessed on the basis of the presence or otherwise of patient's information (patient name, age and gender), prescriber's information (name of the prescriber, contact detail, qualifications, registration number, and signature) and date of consultation. If the full name of the patient or the name with initials was present, that was categorized as the presence of the name or if half of the name was present it was categorized as incomplete name or if the name was not present it was categorized as absence of the patient name. If the age was written with both number and year or months (e.g., 57 years or 5 months), that was categorized as the presence of age, if one component was missing it was an incomplete age and if the age was not mentioned in the prescription it was categorized as absent of age. If the gender of the patient was mentioned it was categorized as presence of gender or otherwise.

Gender is considered present for the purpose of this study if it is mentioned directly or more commonly by the title used in front of the patient's name e.g. Mr., Miss, Mrs.

Prescriber's name, contact details, qualification, registration number and signature were evaluated for their presence or absence. Full name or name with initials was only categorized as presence of the prescriber's name. If part of the name was present, it was categorized as incomplete and if the name was not present it was categorized as absence of the prescriber's name. If the prescriber's address or telephone number or fax number or email was given it was categorized as presence of contact details and at least one from above was not present it was categorized as absence of contact details. If the qualification of the prescriber was present, it was noted down as the prescriber is a specialist or a general practitioner for further data evaluations.

Only the Postgraduate Institute of Medicines accredited specialist was considered as a specialist. Presence or absences of the signature of the prescriber and the registration number were evaluated. Date of consultation was categorized as presence only if the date contains day, month and year. If any component was missing it was an incomplete date. If the date was missing it was categorized as absence of the date. 
The content of the prescription was assessed as the second part of the study. Usage of nonstandard abbreviations was evaluated with the help of the abbreviation list that is mentioned in the British National Formulary [11]. Any abbreviation that is not in that list was categorized as a non-standard abbreviation. Those used non-standard abbreviations were noted down. If units were written as " $\mu \mathrm{g}, \mathrm{ng}, \mathrm{u}$, tsp, or tab" or if there was only numbers without any unit for the strength of the drug, they were categorized as incomplete units. Presence or absences of those were evaluated and if they were present, that abbreviations were noted down. Presence or absences of avoidable decimal points were evaluated.

As the third part of the study, legibility of the prescription was evaluated by three final year B.Pharm students minimizing the error that can occur by not knowing the trade names of the drugs by one person and minimizing being bias by being familiar with one or two prescribers' handwriting.

Each medicine mentioned in the prescription was looked separately for name, dose, frequency and duration. If all 4 categories could clearly be copied, it was labelled as "clear", if all 4 categories could be copied with difficulty, it was labelled as "clear with effort" and if any of the 4 categories could not be copied, it was labelled as "not clear". Then all three assessors' remarks were taken and gave the final categorization using the majority's view (2/3). And if $>75$ of individual components were either clear or clear with difficulty the prescription was labeled as legible and if not illegible. While assessing the legibility of the prescriptions, clarifications of brand names of the drugs were done using the official web site of Cosmetic Drugs and Devices Authority and Sri Lanka Drug Index 2009-2010 [15].

\section{Data analysis}

Microsoft Excel (2010) and Minitab 16 software were used to analyze all the data. Comparisons were made between the variables of the two study locations and between professionals involved in prescription writing (specialists and general practitioners) using Chi square test. The results of incomplete and absent of name of the patient, age, name of the prescriber and date of consultation were combined in the Chi square test considering $p<0.05$ as statistically significant.

\section{RESULTS}

One hundred handwritten outpatient prescriptions from each study location were reviewed for errors in the layout and the content of the prescriptions. Findings related to the studied attributes are shown in Table 1.

According to the Table 1, name of the patient was present in $75(37.5 \%)$ of the prescriptions and age was present in 92 (46\%) of the prescriptions. Name of the prescriber was found in 181 (90.5\%) while 148 (74\%) of the prescriptions had contact details. We found 170 (85\%) and $150(75 \%)$ of prescriptions with the qualifications and signature of the prescriber respectively. Date of consultation was present in $163(81.5 \%)$ prescriptions.

There were 73 (36.5\%) and 102 (51\%) of prescriptions with non-standard abbreviations and incomplete units. Any of the prescription did not include any avoidable decimal point. Among all the prescriptions, we found 99 (49.5\%) legible prescriptions.

Table 1: Percentage occurrence of components of the prescriptions and $p$ values obtained from chi square test for two study locations

\begin{tabular}{lcccc}
\hline Attribute & Frequency (\%) & Aluthgama & Kandy & $\boldsymbol{P}$-value \\
\hline Presence of name of the patient & $75(37.5 \%)$ & 36 & 39 & 0.661 \\
Presence of age & $92(46 \%)$ & 49 & 43 & 0.395 \\
Presence of gender & $155(77.5 \%)$ & 70 & 85 & $0.011^{*}$ \\
Presence of name of the prescriber & $181(90.5 \%)$ & 84 & 97 & $0.002^{*}$ \\
Presence of contact details & $148(74 \%)$ & 64 & 84 & $0.001^{*}$ \\
Presence of qualification & $170(85 \%)$ & 73 & 97 & $0.000^{*}$ \\
Presence of registration number & $32(16 \%)$ & 24 & 8 & $0.002^{*}$ \\
Presence of signature & $150(75 \%)$ & 62 & 88 & $0.000^{*}$ \\
Presence of date of consultation & $163(81.5 \%)$ & 75 & 88 & $0.018^{*}$ \\
Presence of non-standard abbreviations & $73(36.5 \%)$ & 35 & 38 & 0.659 \\
Presence of incomplete units & $102(51 \%)$ & 53 & 49 & 0.572 \\
Presence of avoidable decimal points & $0(0 \%)$ & 0 & 0 & \\
Legible prescriptions & $99(49.5 \%)$ & 48 & 51 & 0.671 \\
\hline
\end{tabular}

${ }^{\star} P<0.05$ is considered statistically significant 
Table 2: $P$-values obtained from Chi square test for specialists and general practitioners

\begin{tabular}{lccc}
\hline Attribute & Specialist & General practitioner & $\boldsymbol{P}$-value \\
\hline Presence of name of the patient & 42 & 12 & $0.000^{*}$ \\
Presence of age & 51 & 47 & 0.572 \\
Presence of gender & 79 & 76 & 0.611 \\
Presence of name of the prescriber & 100 & 100 & 1.000 \\
Presence of contact details & 82 & 82 & $0.000^{*}$ \\
Presence of registration number & 10 & 76 & 0.067 \\
Presence of signature & 82 & 71 & $0.000^{*}$ \\
Presence of date of consultation & 89 & 24 & $0.046^{*}$ \\
Presence of non-standard abbreviations & 37 & 47 & 0.572 \\
Presence of incomplete units & 51 & 0 & \\
Presence of avoidable decimal points & 0 & 65 & $0.022^{*}$ \\
Legible prescriptions & 49 & & \\
\hline
\end{tabular}

${ }^{\star} P<0.05$ is considered as statistically significant

Name of the patient and age was either absent or incomplete in more than $50 \%$ of the prescriptions while all the prescriber details except registration number was present in more than $60 \%$ of the prescriptions in both districts. Nearly half of the prescriptions from Aluthgama and Kandy had incomplete units (Table 1).

Comparison of results between two study locations revealed that results for half of the criteria such as presence of gender of the patient, information of the prescriber and date of consultation are significantly different between those two sites. Resulted chi square values and $p$ values are tabulated in Table 1.

Results of five studied attributes such as name of the patient, date of consultation, non-standard abbreviations and legibility of the prescriptions were significantly different between prescriptions from specialists and general practitioners. Resulted chi square values and $p$ values are tabulated in Table 2.

\section{DISCUSSION}

At the beginning of this research, it was assumed that results from these two locations will be different from each other as they are suburban and urban areas, respectively, where state hospital facilities differ. However, for half of the criteria reviewed, both had approximately equal percentage of errors. Though there is a difference in hospital facilities in these two areas, private consultation centers fill this gap. This may be the reason for having this result.

More than half of the prescriptions from two locations (Table 1) only had a surname or one part of the name of the patient without at least initials as the name which can lead to confusion of the pharmacist and also the patients. As these are outpatient prescriptions which are given to the patient's hand and confusions can happen at home having same surname for the family members. In a study done in India, patient name was absent in $58.5 \%$ of the prescriptions [15] which is similar to the results of this study but a study done in Saudi Arabia reported 94.6\% prescriptions with patient's name [16]. Reported results from an Indonesian study of the presence of age $(52.4 \%)$ which is essential for the clarification of the dose was approximately similar to the results of this study (Table 2) [9]. But similar to the results of the presence of patient's name, same Saudi Arabian study reported $77.2 \%$ prescriptions with patient's age [16]. The reasons for not having complete patient's information in prescriptions from Sri Lanka, India and Indonesia and having that information in prescriptions from Saudi Arabia are probably due to the busy working environment where a prescriber cannot attend to one patient for a longer time and also not having ideal formats for prescriptions.

Information of the prescriber such as prescriber's name, contact details, qualifications and signature were present in more than half of the prescriptions from both places (Table 1). This is because of the usage of rubber seal by the prescribers. But this rubber seal was not clear in most of the prescriptions. Therefore even though contact details are available in the prescription pharmacist may not be able to contact prescribers when it is necessary.

The reported results for the presence of prescriber's information from the Indian study on irrational drug use in India were similar to the results that were revealed by this study [15]. But in the study done in Saudi Arabia, there were more than $80 \%$ prescriptions with prescriber's name and signature [16].

Date of consultation was present in majority of the prescriptions of both locations (Table 1). It 
was approximately similar to the results found from the Indian study where there were $84.5 \%$ prescriptions with date of consultation [15]. But surprisingly prescriptions that were reviewed in the Saudi Arabian study had only $35.7 \%$ prescriptions with the date [16]. This may be due to the difference in the definition of the criteria.

There were two types of non-standard abbreviations in these reviewed prescriptions. They were abbreviations in instructions for drug administration and abbreviation for drug name. Some of the non-standard abbreviations in instructions for drug administration were tsp, tab, $\mathrm{d}, \mathrm{m}, \mathrm{n}$ and $\mathrm{H}$ and abbreviations for drug names were $\mathrm{HCQ}, \mathrm{PCM}$ and $\mathrm{HCT}$. These results which are shown in Table 1 are similar to a Sudan study where there were $37.2 \%$ of prescriptions with non-standard abbreviations [17]. Any of these abbreviations are not standard and they can lead to medication errors. Reason for using abbreviations for drug names can be the scarcity of time and other abbreviations such as tab, $m$ and $n$ may be mostly writing as a habit of the prescriber $[14,18]$.

The occurrence of incomplete units is higher than the occurrence of non-standard abbreviations (Table 1). Mostly apparent incomplete units were $\mu \mathrm{g}, \mathrm{u}, \mathrm{tab}$ and tsp. There were prescriptions with no unit for the strength of the drug and also prescriptions with number of tablets instead of the strength of the drug. These were also taken as incomplete units. These types of incomplete units were written due to the same reasons for writing nonstandard abbreviations. Sometimes negligence of prescriber may lead to prescriptions with no units or write in number of tablets [18]. In a study it is said prescribers leave the strength, dose and quantity of the drugs to decide by the pharmacist [17]. Prescriptions without clear and complete instructions to the pharmacist and to the patient can lead to difficulty in understanding the dosage regimen and adverse effects.

Nearly half of the prescriptions from both locations were illegible as shown in Table 1. Studies done on assessing legibility of the prescription have different definitions for the legibility $[19,20]$. Therefore results may be difficult to compare. A published report from Switzerland states that, legibility was assessed depending on four criteria such as "good, moderate, bad and illegible'. They reported $4 \%$ prescriptions which are illegible [21]. But the study done in Saudi Arabia adopted a method to assess legibility which is similar with our research and found $64.3 \%$ prescriptions which were not clear [16].
Most of the illegible prescriptions which were reviewed had legible drug name while duration and strengths were illegible. It seems like prescribers do not pay attention to the duration and strength of the drug. This was highlighted in a study done on inpatient prescriptions in the United Kingdom where it was said prescribers write only the drug name and left other details such as duration, dose, route and form to complete by house officers [8]. It seems similar to the situation here where prescribers expect to predict those details by pharmacists. Some prescriptions had completely illegible drug names and some had drug names only with legible first letters. This may be due to the fact that prescribers have forgotten full names especially the brand names.

The illegibility will be less if these were assessed using experienced pharmacists or pharmacists from same areas where prescriptions were collected. Because they are more familiar with the brand names and familiar with the particular prescriber's hand writing than final year undergraduate pharmacy students. But here we assumed, a prescription should be legible enough to read by a final year B.Pharm student rather than guessing the drugs in the prescriptions. Another reason for having a high number of illegible prescriptions could be the fact that illegibility of any part from name, dose, frequency and duration of the drug was assessed as illegibility of complete drug.

In the comparison of the two study locations it was revealed that prescribers details are missing significantly more in prescriptions from suburban area compared to the urban area. This might be a regional difference where prescribers are not much concerned about this as there are fewer prescribers. Similarly by comparing specialist and general practitioners, it was revealed that specialists seem to write more name and date of consultation. This is probably because these are written by someone else that is appointed for that task. But legibility of the prescriptions was better with non-specialists and non-standard abbreviations were used more by specialists compared to general practitioners. The registration number of the prescriber was missing more in prescriptions from specialists. This is probably because they think it is not important in view of their specialist qualifications.

\section{Limitations of the study}

The study was conducted in only two communities in two locations, thus limiting the generalizability of the findings. Using one pharmacy in one study location may not be 
representative of the whole area even though the two selected pharmacies were large pharmacies with relatively high number of patients attending daily.

\section{CONCLUSION}

Several areas that need improvement to minimize medication errors are identified in this study and these include stating patient name and age, as well as legibility of prescriptions. Lack of standardization of prescription writing process in relation to layout, use of abbreviations and units and legibility may be the main reason for this unsatisfactory nature of the layout and content of prescriptions. Efforts should be made to enhance the awareness of prescribers, pharmacists and patients regarding prescription errors, and also to stress the necessity of writing prescriptions completely and legibly, preferably in printed form, as well as designing a standard format for prescriptions.

\section{REFERENCES}

1. US Pharmacopeia, United States Pharmacopeia. The Standard. USA: US Pharmacopeia 1995.

2. Anderson P, Townsend T. Medication errors: Don't let them happen to you. Am Nurse Today 2010; 5: 2328.

3. Pote $S$, Tiwari $P$, Cruz $S D$. Medication prescribing errors in a public teaching hospital in India: A prospective study. Pharm Prac 2007; 5: 17-20.

4. Kohn $L T$, Corrigan JM, Donaldson MS. To err is human building a safer health system. Washington, DC. Ann Franesth Rèanim 1999; 21: 453-487.

5. Karna K, Sharma S, Inamdar S, Bhandari A. Study and evaluation of medication errors in a tertiary care teaching hospital - a baseline study. Int J Pharm Pharm Sci 2012; 4: 587-593.

6. Marwaha M, Marwaha RK, Padi JW. A retrospective analysis on a survey of handwritten prescription errors in general practice. Int $J$ Pharm Pharm Sci 2010; 2: 80-82.

7. Maxwell S. Good prescribing: better systems and prescribers needed. Can Med Assoc J 2010; 182: 540-541.
8. Dean B, Barber N, Schachter M. What is a prescribing error? Qual Health Care 2000; 9: 232-237.

9. Perwitasari DA, Abror J, Wahyuningsih I. Medication errors in outpatients of a government hospital in Yogyakarta Indonesia. Int J Pharm Sci Rev Res 2010; 1: 8-10.

10. Ross LM, Wallace J, Paton JY. Medication errors in a paediatric teaching hospital in the UK: five years operational experience. Arch Dis Child 2000; 83: 492-497.

11. British National Formulary (BNF), British Medical Journal Publishing Group and Pharmaceutical Press, United Kingdom 2013.

12. Lehmann CU, Kim GR. Prevention of medication errors. Clin Perinatol 2005; 32: 107-123.

13. Kaushal R, Bates DW, Landrigan C. Medication errors and adverse drug events in pediatric inpatients. JAMA 2001; 285: 2114-2120.

14. Wong IC, Ghaleb MA, Franklin BD, Barber N. Incidence and nature of dosing errors in paediatric medications: a systematic review. Drug Saf 2004; 27: 661-670.

15. Patel V, Vaidya R, Naik D, Borker P. Irrational drug use in India: a prescription survey from Goa. J Postgrad Med 2005; 51: 9-12.

16. Irshaid YM, Homrany MAl, Hamdi AA, Mahfouz AA. Compliance with good practice in prescription writing at outpatient. East Mediterr Health J 2005; 11: 922928.

17. Yousif MA, Nizar S, Elmustafa MO, Mustafa MI, Bella $M M$. Investigation of medication prescribing errors in Wad Medani, Gezira, Sudan. Int J Risk Saf Med 2011; 23: 11-17.

18. Dean B, Schachter M, Vincent C, Barber N. Causes of prescribing errors in hospital inpatients: a prospective study. Lancet 2002; 359: 1373-1378.

19. Auta A, Shalkur D, Banwat SB, Dayom DW. Readability of malaria medicine information leaflets in Nigeria. Trop J Pharm Res 2011; 10(5): 631-635.

20. Akoria $O A$, Isah AO. A comparison of two instruments for the assessment of legibility of prescriptions in a developing country. Trop J Pharm Res 2009; 8(6): 485-489.

21. Hartel MJ, Staub LP, Röder C, Eggli S. High incidence of medication documentation errors in a Swiss university hospital due to the handwritten prescription process. BMC Health Serv Res 2011; 11: 32-38. 